\title{
Heavy-Tailed Statistics in Short-Message Communication *
}

\author{
HONG Wei(洪伟) $)^{1}$, HAN Xiao-Pu(韩篠璞 $)^{1 * *}$, ZHOU Tao(周涛) $)^{1,2}$, WANG Bing-Hong(汪秉宏 $)^{1,3 * * *}$ \\ ${ }^{1}$ Department of Modern Physics, University of Science and Technology of China, Hefei 230026 \\ ${ }^{2}$ Department of Physics, University of Fribourg, CH-1700 Fribourg, Switzerland \\ ${ }^{3}$ Shanghai Academy for Systemic Sciences, Shanghai 200093
}

\begin{abstract}
Short-message (SM) is one of the most frequently used communication channels in modern society. Based on the SM communication records provided by some volunteers, we investigate the statistics of SM communication pattern, including the inter-event time distributions between two consecutive short messages and two conversations, and the distribution of message number contained in a complete conversation. In the individual level, the empirical result raises strong evidence that the human activity pattern, exhibiting a heavy-tailed inter-event time distribution, is driven by a non-Poisson process.
\end{abstract}

Information communication builds the basis of social relations. In the modern science, statistical analysis on the communication database becomes one of the most important approaches to reveal the social structure. ${ }^{1]}$ For example, the communication structures based on E-mail ${ }^{[2]}$ and phone-call ${ }^{[3]}$ display scale-free and small-world properties, which are ubiquitous in various social networks. For the lack of long-term standard database about human communication activities, previous studies on social communication systems usually simply assume the temporal occurrence of contacts between two people is uniform. That is to say, given two nodes in a social acquaintance network, at any time the occurring probability of a new contact (e.g. telephone call, SM communication, on-line instant chat, e-mail communication, etc.) is the same, which leads to a Poisson distribution of inter-event time between two consecutive contacts. However, recently the empirical investigations on e-mail ${ }^{[4]}$ and surface mail ${ }^{[5]}$ communication show a far different scenario: those communication patterns follow non-Poisson statistics, characterized by bursts of rapidly occurring events separated by long gaps. That is, the inter-event time distribution has a much fatter tail than the exponential form, approximated to a power law. The similar statistical properties have also been found in many other human behaviours, ${ }^{[6]}$ including market transaction, ${ }^{[7-9]}$ on-line game playing, ${ }^{[10]}$ movie watching, ${ }^{[11]}$ web browsing, ${ }^{[12]}$ music listening, ${ }^{[13]}$ computer operations ${ }^{[14]}$ and so on. Those empirical statistics clearly indicate the invalidity of Poisson process in mimicking the human dynamics in many reallife systems. Motivated by those empirical evidences, scientists are desired to uncover the origin of heavytails in human dynamics, as well as to reveal the effect of non-Poisson statistics on some dynamical process in social systems. In the former aspect, Barabási et al. suggested the highest-priority-first (HPF) protocol a potential origin, $\left.{ }^{4}{ }^{4} 15\right]$ however, this queuing model may not well explain all the possible mechanisms leading to a heavy tail. ${ }^{[16]}$ In the latter aspect, so far only few works about epidemic spreading are reported. ${ }^{[17]}$

Based on the rapid progress on wireless techniques, the SM communication becomes one of the most important social contact tools in the modern society. Recent studies based on the mobile communications discovered the scaling law in human travels. ${ }^{[18]}$ In this Letter, based on the SM communication records provided by some volunteers, we investigate the statistics of SM communication pattern, including the inter-event time distributions between two consecutive short messages and two conversations, and the distribution of message number contained by a complete conversation. In the individual level, the empirical result raises a strong evidence that the human activity pattern, exhibiting a heavy-tailed inter-event time distribution, is driven by a non-Poisson nature.

In this study, the SM records of eight volunteers (signed from A to $\mathrm{H}$ ) are investigated. Those contain one company manager (C) and seven University students (else). The overall time spans of those records range from three to six months. Every record contains the sending time of SMs, and the records A, C, E, F contain the cell phone numbers of the receivers. Some basic properties of the records are listed in Table 1.

We focus on the common properties shared by different records, because those may imply some general statistical characteristics of the human temporal activities. The time is coarse-grained in an hour resolution.

We present the empirical results of (i) the inter-

*Supported by the National Natural Science Foundation of China under Grant Nos 70871082,10635040 and 10532060 , the National Basic Research Programme of China under Grant No 2006CB705500, and the National Science Fund for Fostering Talents in Basic Sciences (J0630319).

** Email: hxp@mail.ustc.edu.cn

*** Email: bhwang@ustc.edu.cn 
event time distribution of sending SMs, (ii) the distribution of inter-event time between two consecutive conversations, and (iii) the length distribution of conversations. The results indicate that the communication behaviours of different users share some common properties, especially the observed heavy tails in all those distributions.

Table 1. Properties of the records provided by the volunteers.

\begin{tabular}{cccc}
\hline Records & $\begin{array}{c}\text { Time span } \\
(\text { month })\end{array}$ & $\begin{array}{c}\text { Total number } \\
\text { of SMs }\end{array}$ & $\begin{array}{c}\text { Average number of } \\
\text { SMs sent per day }\end{array}$ \\
\hline A & 6 & 1528 & 8.7 \\
B & 6 & 4844 & 26.8 \\
C & 6 & 5987 & 33.6 \\
D & 3 & 3780 & 41.1 \\
E & 6 & $4523\left(2263^{a}\right)$ & 25.4 \\
F & 4.5 & 5778 & 41.6 \\
G & 5 & 5346 & 34.9 \\
H & 5.5 & 3734 & 22.1 \\
\hline${ }^{a}$ Number of SMs recording the cell phone numbers of
\end{tabular}
receivers.
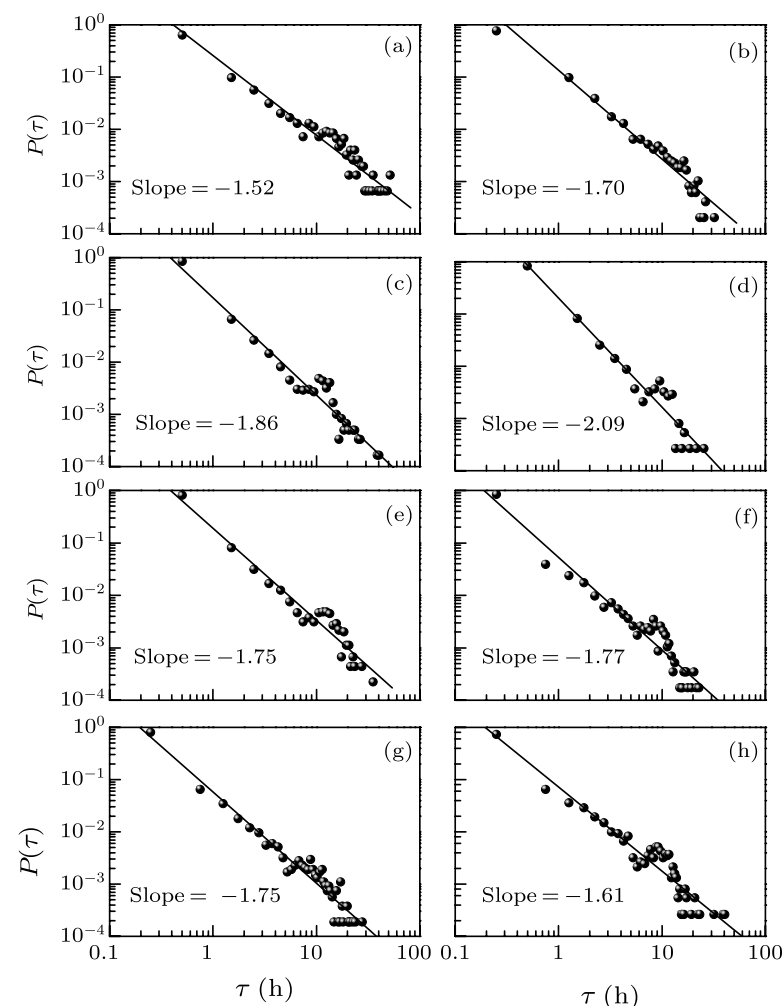

Fig. 1. The log-log plots of inter-event time distributions. The black dots represent the empirical data, and the red lines are the linear fittings. The panels (a)-(h) correspond to the records of $\mathrm{A}-\mathrm{H}$, respectively.

As shown in Fig. 1, the inter-event distribution can be well fitted by a power-law form $P(\tau) \propto \tau^{-\alpha}$, where the exponent $\alpha$ is between 1.5 and 2.1 for different users. In each curve, an obvious peak turns up at about ten hours, which is related to the physiological period of human: the sleeping time are more or less ten hours. In addition, as shown in Fig. 2, there are obvious positive correlation between the average numbers of SMs sent per day and the exponent $\alpha$. This phenomenon, similar to the observations in the on-line movie watching, ${ }^{[11]}$ is against the hypothesis ${ }^{[15]}$ on the discrete universality classes of human dynamics.

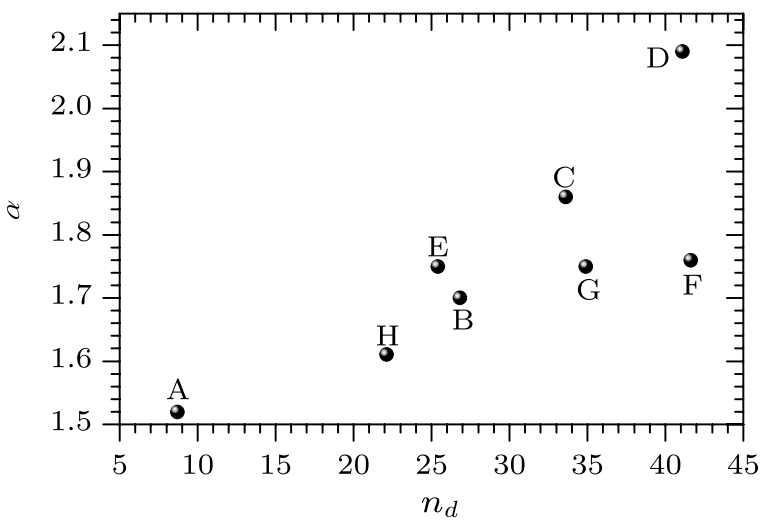

Fig. 2. Dependence between the exponent $\alpha$ of each distribution and its daily average number $n_{d}$.
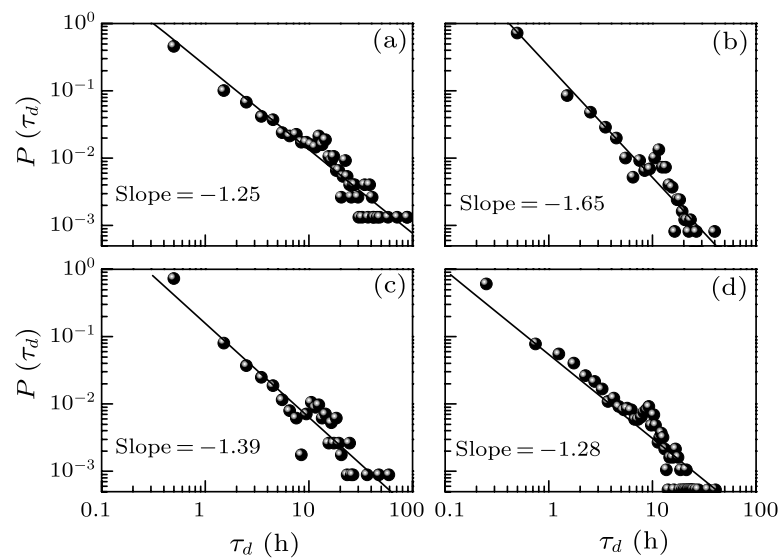

Fig. 3. Inter-event time distributions of conversations in log-log plots. The black dots represent empirical data, and the lines are the linear fittings, with (a), (b), (c) and (d) corresponding to the records of $\mathrm{A}, \mathrm{C}, \mathrm{E}$ and $\mathrm{F}$, respectively.

Generally speaking, in the SM communication, a user often consecutively sends/receives messages to/from one person. According to our daily experience, when people are engaged in the SM communication, they often need several times of exchanging messages to build a complete conversation. Therefore, we define a conversation as SMs that are sent to one person consecutively, without being interrupted by some other persons. Furthermore, the inter-event time between two consecutive conversations is defined as the time difference between the two beginning times. Only the samples A, C, E and F are investigated since their records contain the phone numbers of receivers. As shown in Fig. 3, the inter-event time distributions of conversations can be well fitted by power laws, and the values of exponents are less than the corresponding distributions of SM sending. Actually, the conversation rather than a single SM can better characterize the communication pattern because a conversation is functional complete. Yet it should be reminded that, in the real communication process, one complete con- 
versation may be interrupted by other correspondents, so that a single conversation may be regarded as several small ones. Since these conditions cannot be automatically discriminated from the empirical data, they may cause some bias to a certain extent. However, the statistical characteristics such as heavy tails shown in Fig. 3 are believable, because the range of the distribution will be enlarged and the tail of the distribution will be even fatter if the bias is eliminated. To characterize the strength of a conversation, we define a length of a conversation as the number of SMs it contains. As shown in Fig. 4, every length distribution is dominated by a power law with exponent larger than 2 .

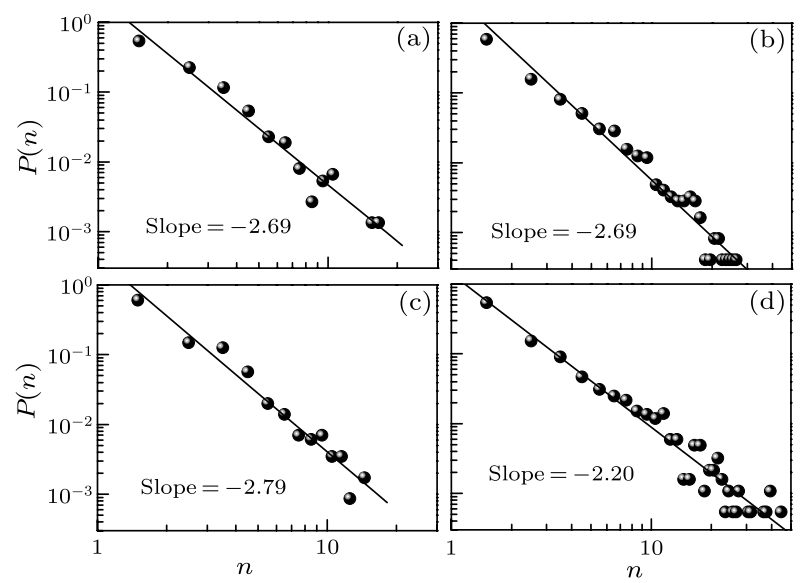

Fig. 4. The log-log plots of length distributions of conversations. The black dots represent empirical data, and the lines are the linear fittings, with (a), (b), (c) and (d) corresponding to the records of $\mathrm{A}, \mathrm{C}, \mathrm{E}$ and $\mathrm{F}$, respectively.

In summary, we have investigated some statistical properties of SM communication in the individual level. The empirical evidence indicates that the SM communication pattern is governed by a non-Poisson statistics. For commercial reason and the right of personal privacy, we could not freely and automatically download the SM data without permission. Therefore, those results are limited by the lack of data. In despite of the small number of samples, all the records display very similar statistics, thus we believe those findings shown in this study are common for the most of SM users.

The temporal statistics in SM communication are similar to those observed in e-mail ${ }^{[4]}$ and surface mail ${ }^{[5]}$ communications. Besides their similarity, the SM communication has some specific features different from e-mail and surface mail. Firstly, it is directly perceived through the senses to treat surface mails as some tasks waiting for reply. We may soon reply the urgent and important letters, and the ones not important or difficult to reply may wait for a long time before replied. In contrast, we usually reply a short message immediately. It is seldom seen that a short message is very hard to reply, thus we have to spend a long time in preparing the response. Therefore, instead of the HPF protocol, ${ }^{[4]}$ the heavy-tailed distribution may root in some other mechanisms, such as the competition with other tasks, ${ }^{[15]}$ the personal interest, ${ }^{[16]}$ the social interactions among many users, ${ }^{[19]}$ and so on. Secondly, a single short message is usually a tiny part of a complete conversation, thus to study the statistics in the resolution of conversation may be more proper to reflect the function of SM communication.

Based on the analytical solution ${ }^{[20]}$ of the Barabási model, ${ }^{[4]}$ Vázquez et al. ${ }^{[15]}$ claimed the existence of two discrete universality classes of human dynamics, whose characteristic power-law exponents are 1 and 1.5, respectively. The e-mail communication, web browsing and library loans belong to the former, while the surface mail communication belongs to the latter. However, so far there has been increasing empirical evidence against the hypothesis of universality classes for human dynamics. ${ }^{[6]}$ As shown in Fig. 1, different individuals have different power-law exponents which are, typically, larger than 1.5. Furthermore, Fig. 2 show a positive correlation between activity and power-law exponent, which is against the discrete universality classes. However, we also note that the power-law exponents in the inter-event distributions of conversations are closer to 1.5. A clearer picture asks for abundant data in the future.

The authors thank Shuang-Xing Dai and GuanXiong Chen for valuable discussion, as well as LingZhi Hu and Kai Pan for the assistance of manuscript preparation.

\section{References}

[1] Watts D J 2007 Nature 445489

[2] Eckmann J P, Moses E and Sergi D 2004 Proc. Natl. Acad. Sci. U.S.A. 10114333

[3] Aiello W, Chung F and Lu L 2000 Proceedings of the 32nd ACM Symposium on the Theory of Computing (New York: $\mathrm{ACM})$ p 171

[4] Barabási A L 2005 Nature 435207

[5] Oliveira J G and Barabási A L 2005 Nature 4371251 Li N N, Zhang N and Zhou T 2008 Physica A 3876391

[6] Zhou T, Han X P and Wang B H 2008 Science Matters: Humanities as Complex Systems ed Burguete M and Lam L (Singapore: World Scientific) p 207

[7] Politi M and Scalas E 2008 Physica A 3872025

[8] Jiang Z Q et al 2008 Physica A $\mathbf{3 8 7} 5818$

[9] Masoliver J et al 2003 Phys. Rev. E 67021112

[10] Henderson T and Nhatti S 2001 Proc. 9th ACM Int. Conf. on Multimetia (New York: ACM) p 212

[11] Zhou T et al 2008 Europhys. Lett. 8228002

[12] Dezsö Z et al 2006 Phys. Rev. E 73066132 Goncalves B and Ramasco J J 2008 Phys. Rev. E 78026123

[13] Hu H B and Han D Y 2008 Physica A 3875916

[14] Baek S K, Kim T Y and Kim B J 2008 Physica A 3873660

[15] Vázquez A et al 2006 Phys. Rev. E 73036127

[16] Han X P et al 2008 New J. Phys. 10073010

[17] Vázquez A et al 2007 Phys. Rev. Lett. 98158702

[18] Gonzalez M C et al 2008 Nature 453779

[19] Oliveira J G and and Vázquez A arXiv: 0710.4916

[20] Vázquez A 2005 Phys. Rev. Lett. 95248701 